The International Journal of Engineering and Science (IJES)

|| Volume || 6 || Issue || 7 || Pages || PP 01-12 || 2017 ||

ISSN (e): 2319 - 1813 ISSN (p): $2319-1805$

\title{
Comparison of Heat Transfer rate of closed loop micro pulsating heat pipes having different number of turns
}

\author{
Malay S. Patel ${ }^{1}$, Sulochan D. Mane ${ }^{2}$, Sandeep S. Mopare ${ }^{3}$, Dhananjay Y. Patil ${ }^{4}$ \\ 1,2,3,4 Department of Mechanical Engineering, Dr. D. Y. Patil Institute of Engineering and Technology, Pimpri, \\ Pune, India \\ Email:patelmalay68@gmail.com,sulochanmane77@gmail.com,sandeepmopare0496@gmail.com, \\ pdhananjay0@gmail.com
}

ABSTRACT-

In the present study the heat transfer capacity of the micro pulsating heat pipes. The fundamentals of working of the Micro Pulsating Heat pipes (MPHP) are explained. The micro pulsating heat pipes having channel diameter $800 \mu \mathrm{m}$ and different number of turns have been designed. These MPHPs are filled with water. Different numbers of turns have been designed. Different filling ratios i.e. 30\%, 50\%, 70\% have been selected for the MPHPs. On each case a constant heat flux of $1000 \mathrm{~W} / \mathrm{m}^{2}$ has been applied. The heat transfer rate is found out in each case and compared to find out the optimum case. All the simulation work has been carried out in Ansys Fluent.

Keywords: Micro pulsating Heat Pipe, Filling ratio, Critical Diameter, Heat Transfer rate, Thermal analysis of heat pipe, Volume Fraction.

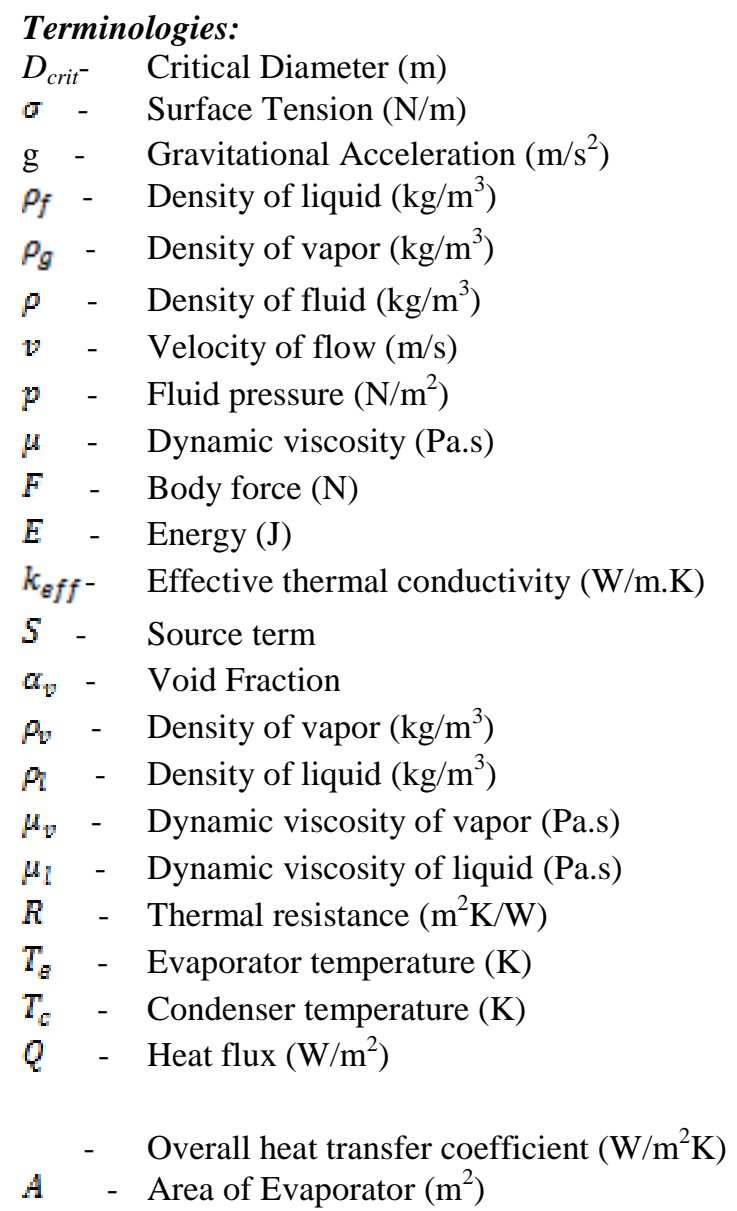




\section{INTRODUCTION}

Micro Pulsating Heat Pipes also known as open loop or closed loop oscillating Heat pipes in the literature, possess outstanding heat transfer performance and are increasingly used in the area of electronics and cooling. With the development of semiconductor, the electronic devices are getting increasingly heated in the recent years. As the devices become more densely packed the heat flux generated is of high magnitude. So, a cooling device which is compact in size, having excellent heat transfer capacity has to be developed that well provide cooling.The heat pipe is a very good example of such device. But, Heat pipe have limitation that they can be made thin and flexible only up to certain limit. Also the use of wick inside them makes them less suitable for smaller devices. So, an MPHP that can be of open loop(OL) or closed loop(CL) for is mostly used [3]. They are of serpentine shape and do not have wick structure inside them.

\subsection{Literature Review}

1) 'CFD and volume fraction analysis of LPHP' by Mr. Mohd Aijaz al Haque and Mr. Md Azizuddin. In this paper the authors have done the CFD and volume fraction analysis of single turn CLPHP. There are different configurations of copper capillary tubes having lengths $540 \mathrm{~mm}$ and diameters $1.95 \mathrm{~mm} w e r e$ taken and were filled with filling ratio of $60 \%$ and $80 \%$. The heat flux was varied and the heat transfer rate was found in each case and was compared with the experimental result. Certain theoretical concepts with formulae of this paper have been included in the present study.

2) 'Comparison of the thermal performances and flow characteristics between closed loop and closed end micro pulsating heat pipe.' By Sooh wan Jun and Sung Jin Kim, in this paper experiments were performed to find which type of micro pulsating heat pipe would perform better between CLMPHP and Closed End Micro Pulsating Heat Pipe. Through thermometry and photography by varying input powers and inclination angles for MPHPs with 5, 10,15 and 20 turns the thermal performance between the two types were compared. In this case of 20 turns it was found that the CLMPHP and CEMPHP in vertical orientation and up to 2.5 times in the horizontal direction. Some theory and figures from this paper have been included.

3) 'Microgravity Performance of Micro Pulsating Heat Pipes' by Junji Gu and Masahiro Kawaji. In this paper an MPHP made of Teflon of $1.6 \mathrm{~mm}$ inner diameter was used to observe the pulsating flow under different gravity levels. Under normal and hyper gravity condition, both the orientation of the pulsating heat pipe and the locations of the heat pipes and the cooling sections affected the heat transfer performance. Under microgravity the heat pipes showed better operating and heat transfer performance than that under normal and hyper gravity. Certain Information from the introduction has been included from this paper.

4) 'Analysis of Chaotic Flow in 2D Multi-Turn Closed Loop Pulsating Heat Pipe' by S.M. Pouryoussefi and Yuwen Zhang. In this paper numerical study has been conducted for the chaotic low in multi turn closed loop pulsating heat pipe. Water was used as the working fluid. Heat flux and constant temperature were used as boundary conditions for evaporator and condenser sections respectively. An optimal filling ratio and minimum thermal resistance were found for better thermal performance of the pulsating heat pipe. Equations for the governing the performance of the heat pipe has been taken from this paper

\subsection{Working Principle of Micro Pulsating Heat Pipe}

MPHP are heat pipes that do not have wick structure and instead contain a rectangular micro channel forming a closed loop. The heat gets transferred from the evaporator to the condenser by means of an axial oscillation of liquid slugs and vapor slugs. The MPHP has many advantages including high thermal performance and maximum effective thermal conductivity of $600 \mathrm{~W} / \mathrm{mK}$ which is 3.5 times higher than that of silicon and 1.5 times that of copper. It is possible to make a small, thin and flat structure and if a flexible material is used for the base and cover materials it is possible to make a flexible Micro Pulsating Heat Pipe because there is no wick structure [5]. 


\subsection{Fabrication of MPHP}

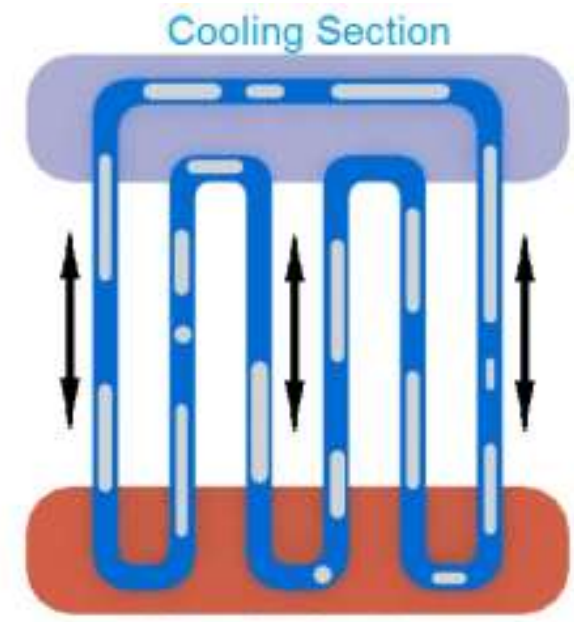

Heating Section

Fig 1: Sectional view of MPHP [5]

The MPHP is fabricated by MEMS techniques. The base material used is silicon. The fabrication is completed by bonding the top cover plate and filling the pipe with the working fluid. The tube diameter is between 0.1 to 5 mm.

The gases in the working fluid are removed via degassing process 3 times to minimize the effect of noncondensable gases. This is done as follows. The working fluid is boiled for more than 30 minutes and then completely frozen by liquid nitrogen. Then the chamber containing the working fluid is evacuated using rotary vane vacuum pump. Then the liquid is filled inside the MPHP [3].

\subsection{Comparison between traditional Heat pipe and MPHP}

Table 1. Comparison between traditional HP and MPHP

\begin{tabular}{|l|l|l|}
\hline Parameter & Traditional HP & MPHP \\
\hline Working Principle & Capillary Effect & Oscillating flow \\
\hline Design and building & Difficult & Easy \\
\hline Space requirement & more & less \\
\hline Pressure drop & high & small \\
\hline Heat transfer capacity & More & less \\
\hline Cost & more & less \\
\hline
\end{tabular}

\subsection{Governing formulae for MPHP}

Critical Diameter of MPHP is the maximum hydraulic diameter in order to form slug train in MPHP [1]. It is given by

$$
D_{\text {crit }}=1.84 \sqrt{\frac{\sigma}{g\left(\rho_{f}-\rho_{g}\right)}}
$$

It should be noted that the value of the diameter of the tubes of MPHP should be less than critical diameter for slug flow to be occurred.

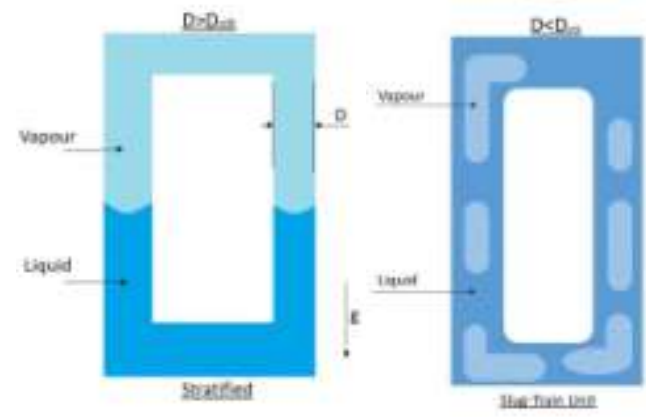

Fig 2. Significance of Critical Diameter [5]

The momentum equation is the basic equation which is used to analyze the flow and is dependent on the volume fractions of all the phases through the properties of density and dynamic viscosity [2]. It is given by 


$$
\frac{\partial}{\partial t}(\rho \vec{v})+\nabla \cdot(\rho v v)=-\nabla p+\nabla \cdot\left[\mu\left(\nabla v+\nabla v^{T}\right)\right]+\rho g+F
$$

The energy equation also shared among the phases is given by [2]

$\frac{\partial}{\partial t}(\rho E)+\nabla \cdot(v(\rho E+\rho))=\nabla \cdot\left(k_{\text {eff }} \nabla T\right)+S$

In the vapor liquid two phase system the density and viscosity in each cell is given by [2]

$$
\rho=\alpha_{v} \rho_{v}+\left(1-\alpha_{v}\right) \rho_{l}
$$

$\mu=\alpha_{v} \mu_{v}+\left(1-\alpha_{v}\right) \mu_{l}$

The thermal resistance which is used as a thermal performance index is estimated by the heat input and the temperature difference between the evaporator and condenser [3].

$R=\frac{T_{Q}-T_{C}}{Q}$

The thermal performance is also found out by calculating the overall heat transfer coefficient. It is inversely proportional to thermal resistance [1]. It is given by

$$
=\frac{Q}{A\left(T_{Q}-T_{R}\right)}
$$

\section{MODELING OF MPHP}

In this paper two dimensional modeling of micro pulsating heat pipe has been done with different number of turns namely 1, 2 and 3. Also different filling ratios 30\%,50\%, 70\% have been experimented and the heat transfer rate is found out in each case.

Following are the geometries of the models of the MPHPs which have been considered for the study.

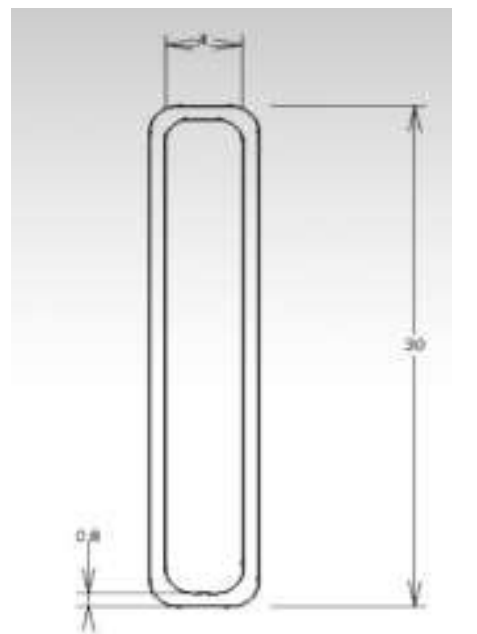

Fig 2. Single turn MPHP geometry

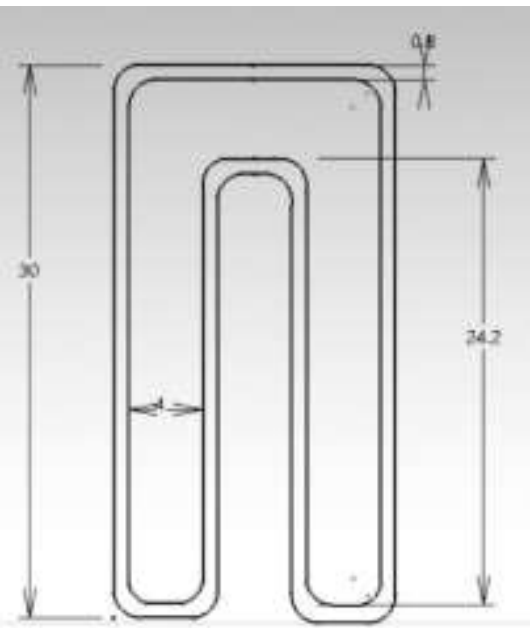


Fig 3. Two turns MPHP geometry

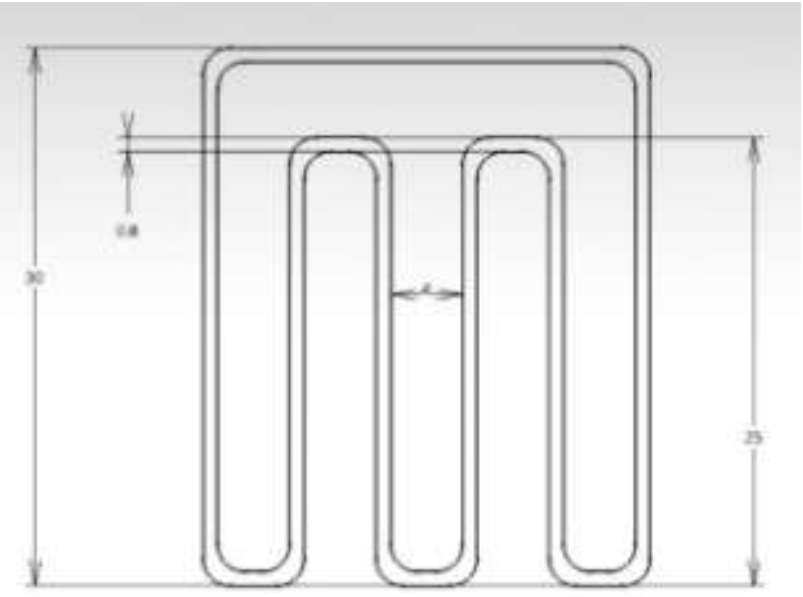

Fig 4. Geometry of three turn MPHP

Design Specifications of the MPHP are as follows

Table 2. Design Specifications of MPHP

\begin{tabular}{|c|c|c|c|c|}
\hline $\begin{array}{c}\text { No of } \\
\text { Loops }\end{array}$ & $\begin{array}{c}\text { Width of channel } \\
(\mathrm{mm})\end{array}$ & $\begin{array}{c}\text { Length of Evaporator } \\
\text { section }\end{array}$ & $\begin{array}{c}\text { Length of condenser } \\
\text { section }(\mathrm{mm})\end{array}$ & $\begin{array}{c}\text { Length of } \\
\text { Adiabatic section }\end{array}$ \\
\hline 1 & 0.8 & 18 & 18 & 32 \\
\hline 2 & 0.8 & 34.4 & 39.6 & 56 \\
\hline 3 & 0.8 & 60 & 59.2 & 84 \\
\hline
\end{tabular}

\subsection{Meshed models of MPHP}

After modeling the next step carried out was meshing. Meshing is nothing but conversion of a model having infinite elements into finite elements. So the physics preference of meshing was 'mechanical' and the curvature was kept on. The smoothing was medium. The meshing was deliberately made like this because otherwise the time taken to solve the model becomes very large and the courant number can spike at any time. The number of elements for single, double and triple turn MPHP's were 302,469 and 879 respectively and the number of nodes were 439,727 and 1298 respectively. The shape of the elements was 'quadrilateral'. Below are the meshed models.

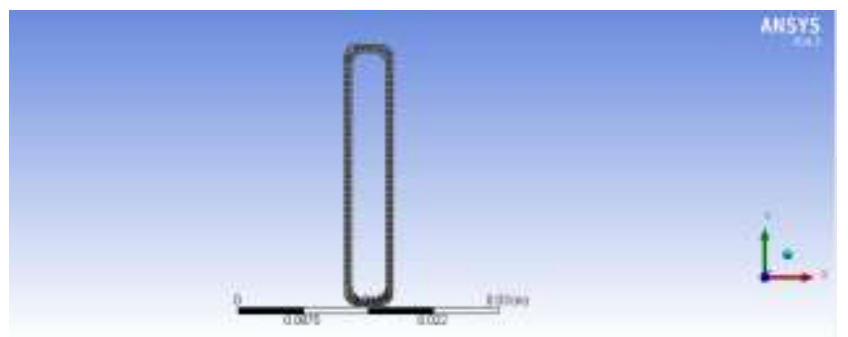

Fig 5. Meshed model of single turn MPHP

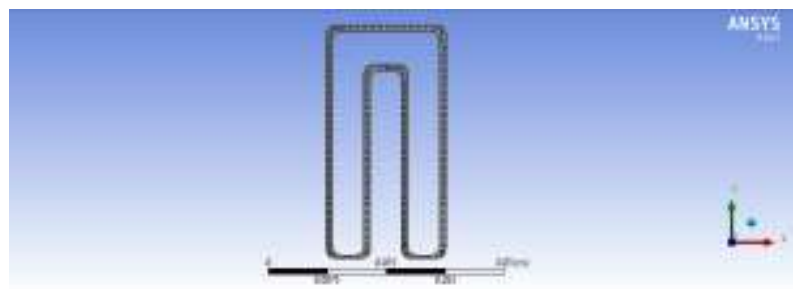

Fig 6. Meshed model of two turn MPHP 


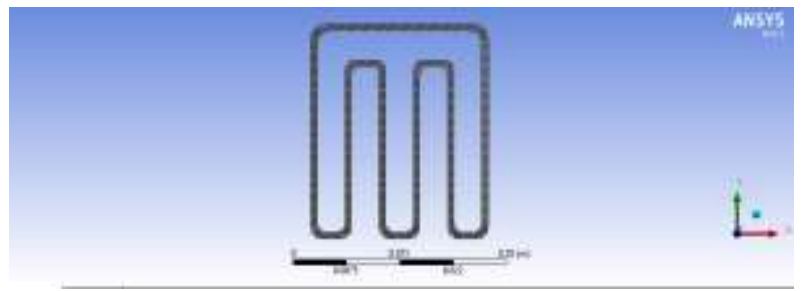

Fig 7. Meshed model of three turn MPHP

\subsection{Initial conditions for solving models}

The models of MPHP were solved in Ansys Fluent using Computational Fluid Dynamics (CFD). CFD is a tool to solve engineering problems involving heat transfer and fluid flow. For each type of pipe the heat flux at the evaporator was kept constant and filling ratios were varied to observe the results. The working fluid was taken as water. The material of the pipe was 'Aluminum'. The fluid inside the pipe comprised of three parts namely water vapor, water liquid and air. The heat flux applied at the evaporator section was $1000 \mathrm{~W} /$ and the temperature of the condenser section was set to $300 \mathrm{~K}$. This is because it is the ideal room temperature and the condenser section is always in contact with the surrounding ambient air. The channel width was $0.8 \mathrm{~mm}$. The inside of the pipe was presumed as vacuum with pressure of $4000 \mathrm{~Pa}$. The saturation temperature of water inside the pipe was taken as $308 \mathrm{~K}$. The flow inside each pipe was transient, which means it varied with respect to time. The pipes were assumed as vertical with gravity (g) acting in the negative y-direction. While solving, Volume of Fluid (VOF) option was taken. This is because The flow inside a pulsating heat pipe is called slug flow which is characterized for a liquid-gas flow in which the gas phase exists as large bubbles separated by liquid "slugs". For this kind of flow the VOF (volume of fluid) approach is the most adequate because it tracks the interface of the phases, which is very important to describe this flow [6]. The flow was taken as laminar as Re came out to be 2200 . Thickness of wall was considered as $0.0005 \mathrm{~mm}$. The filling ratios viz. $30 \%$, $50 \%$, and $70 \%$ were adjusted via adjusting the coordinates. The table below gives details of the working fluid which is water.

Table 3. Initial conditions of MPHP

\begin{tabular}{|c|c|c|c|c|}
\hline Description & Symbol & Water liquid & Water vapor & air \\
\hline Density & $\rho$ & 998.2 & 0.5542 & 1.225 \\
\hline Dynamic viscosity & $\mu$ & 0.001003 & 0.0000134 & 0.0000178 \\
\hline Specific heat & $C_{\bar{y}}$ & 4187 & 1996 & 1006.43 \\
\hline Thermal Conductivity & $\mathrm{k}$ & 0.6 & 0.0261 & 0.0242 \\
\hline
\end{tabular}

\subsection{CFD details for the Problem}

For the problem considered, the time step chosen was 0.0005 seconds. This is because the flow cannot be visualized if the time step is too large. The numbers of time steps were 30000 and so the number of iterations came out to be 210000 . It took approximately $5 \mathrm{hrs}$ to solve each case.

\section{RESULTS}

The volume fraction and the temperature contours for each case were found out as follows. The heat transfer rate has also been found out in the subsequent points based on these results.

\subsection{Single turn MPHP analysis \\ 3.1.1 Water liquid volume fraction and Temperature Distribution for $\mathbf{3 0 \%}$}




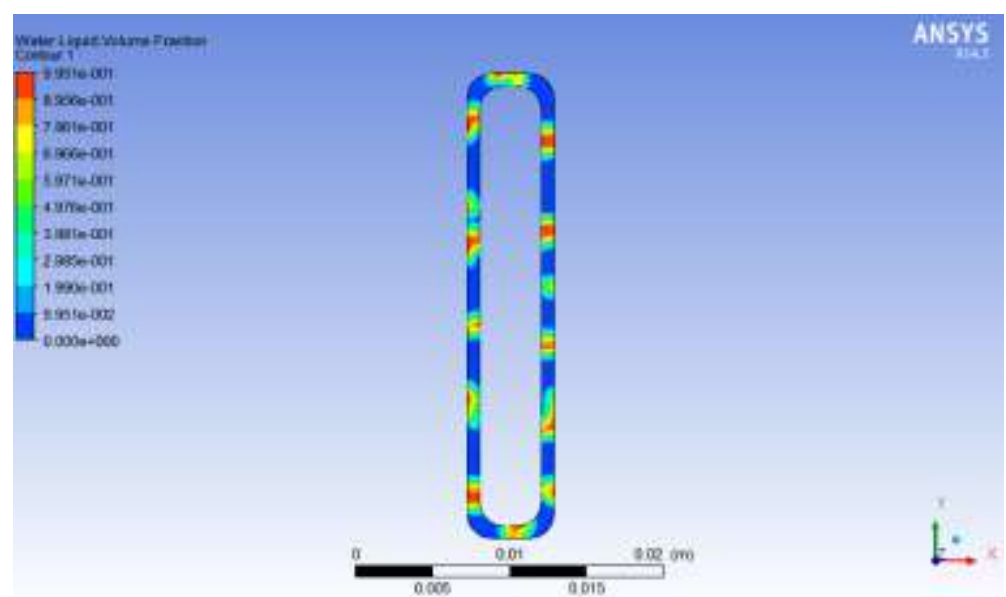

Fig 8. Volume fraction for $30 \%$

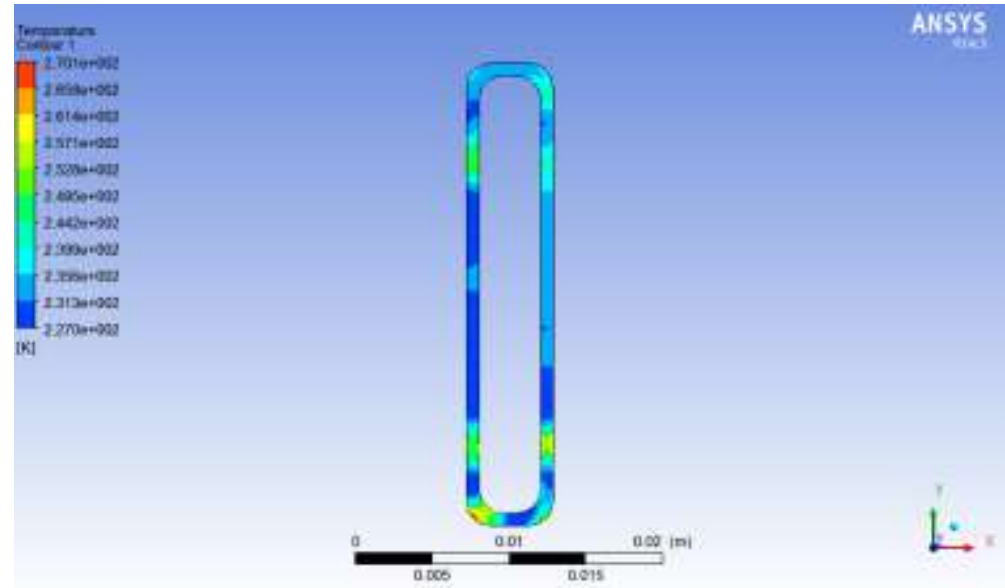

Fig 9. Temperature distribution for $30 \%$

3.1.2 Water volume fraction and Temperature distribution for $\mathbf{5 0 \%}$

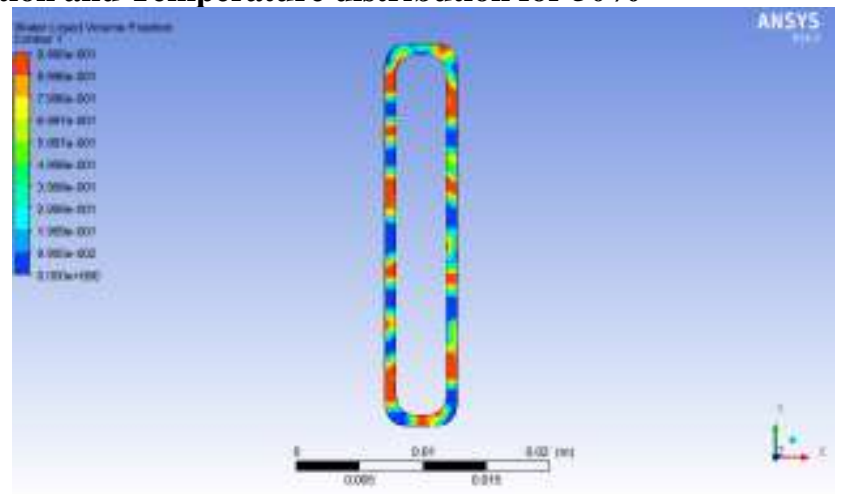

Fig 10. Volume fraction for $50 \%$ 


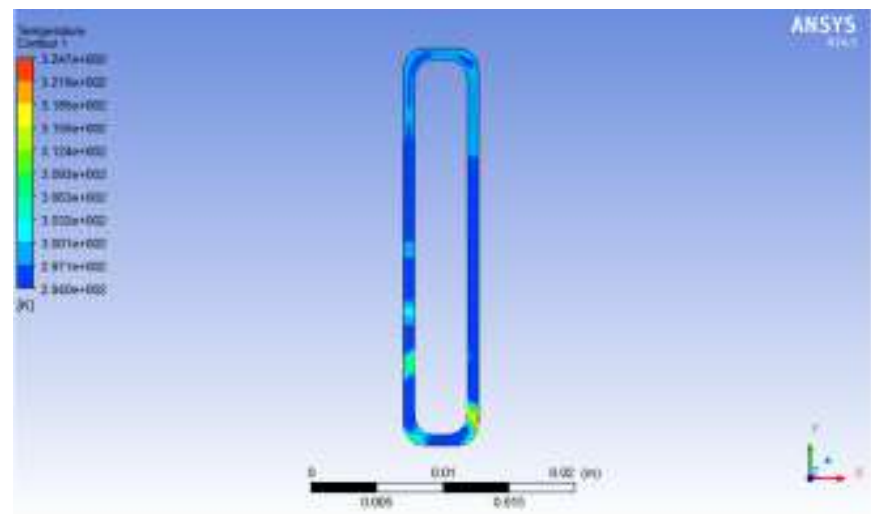

Fig 11. Temperature distribution for $50 \%$

3.1.3 Water volume fraction and Temperature distribution for $\mathbf{7 0 \%}$

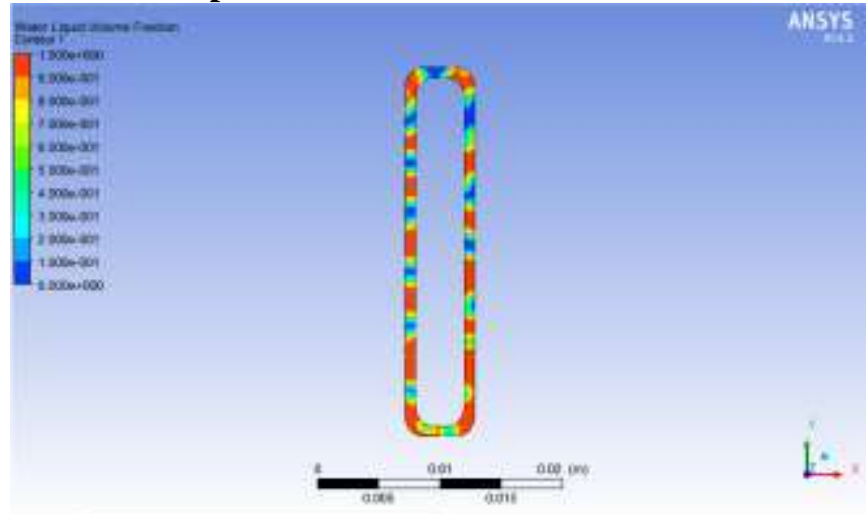

Fig 12. Volume fraction for $70 \%$

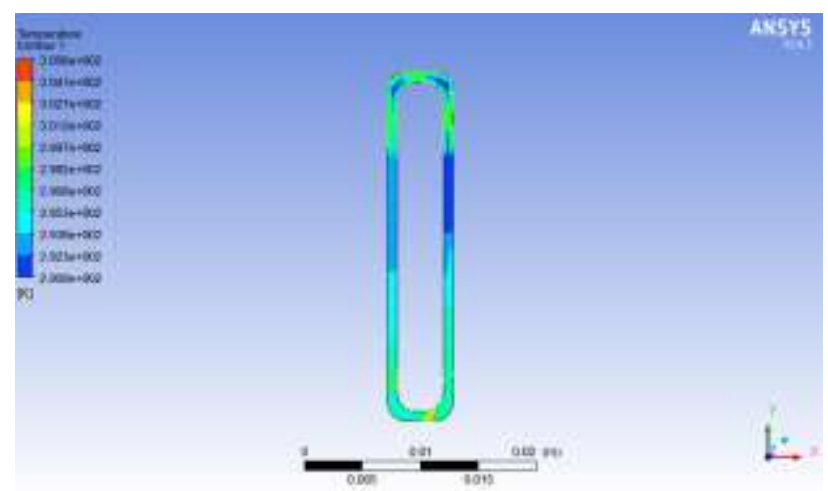

Fig 13. Temperature distribution for $70 \%$

3.2 Two turn MPHP analysis

3.2.1 Water liquid volume fraction and temperature distribution for $30 \%$

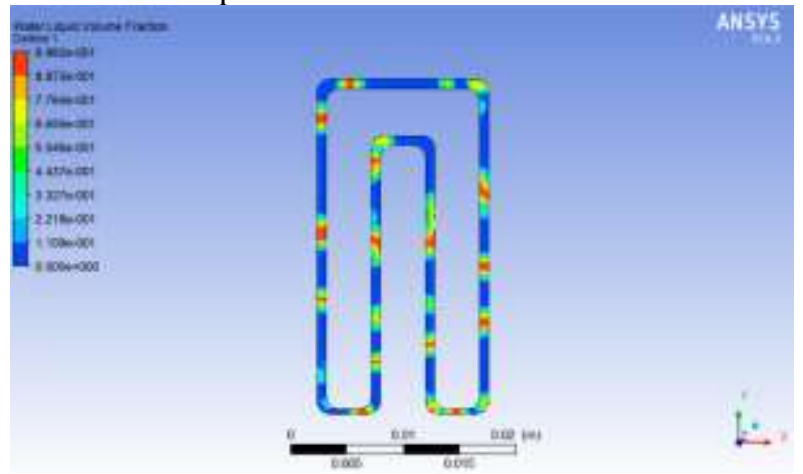

Fig 14. Volume Fraction for $30 \%$ 


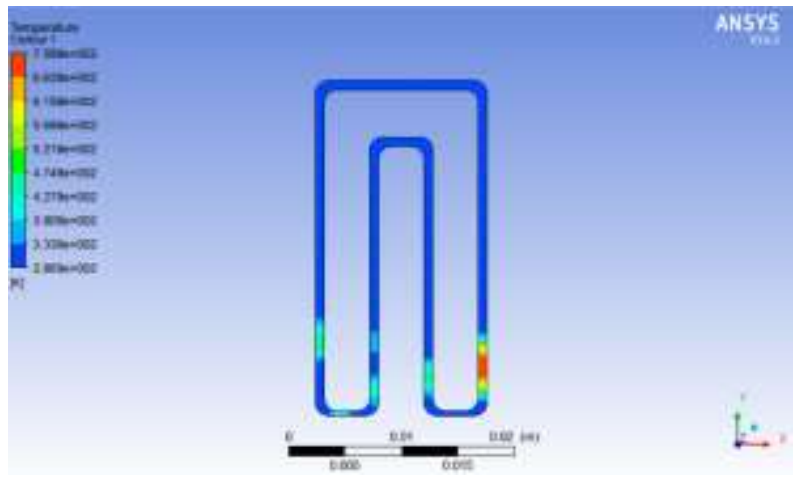

Fig 15. Temperature distribution for $30 \%$

3.2.2 Water liquid volume fraction and temperature distribution for $50 \%$

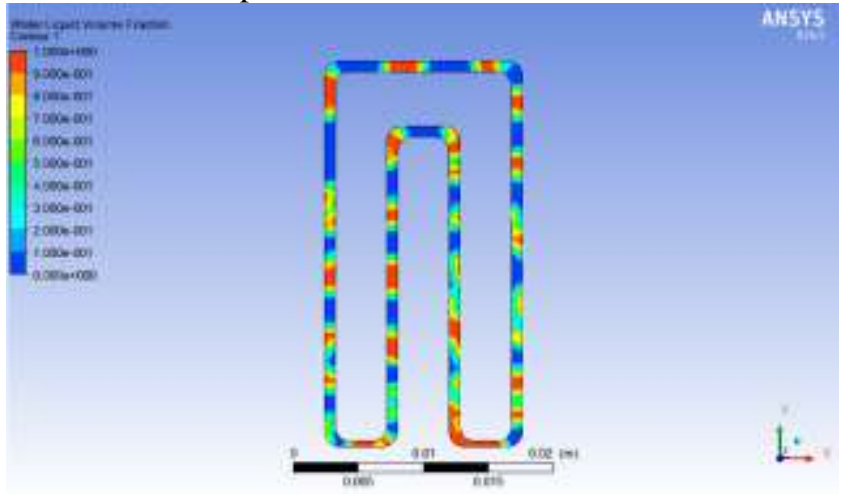

Fig 16. Volume Fraction for 50\%

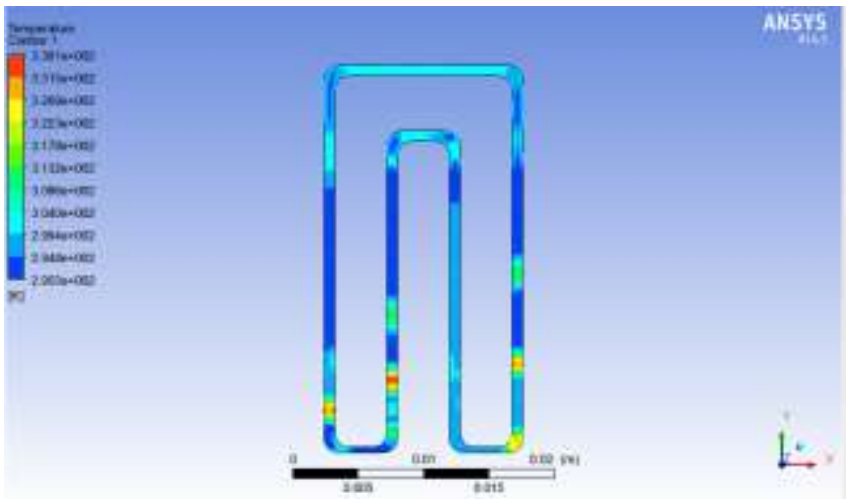

Fig 17. Temperature distribution for $\mathbf{5 0 \%}$

3.2.3 Water liquid volume fraction and temperature distribution for $70 \%$

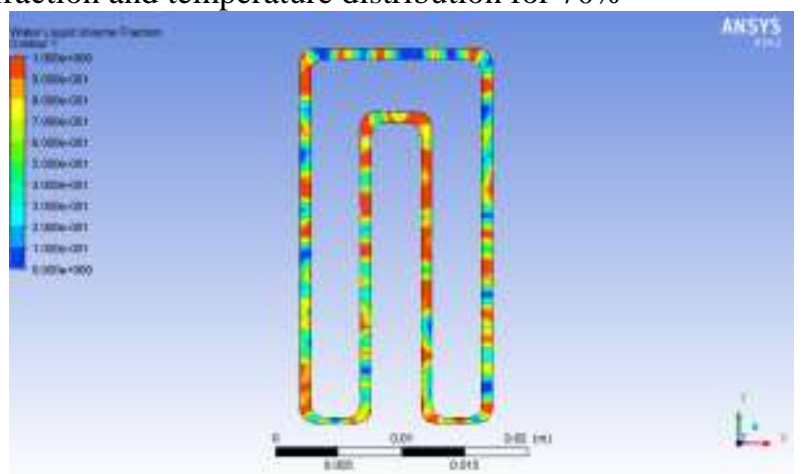

Fig 18. Volume Fraction for $70 \%$ 


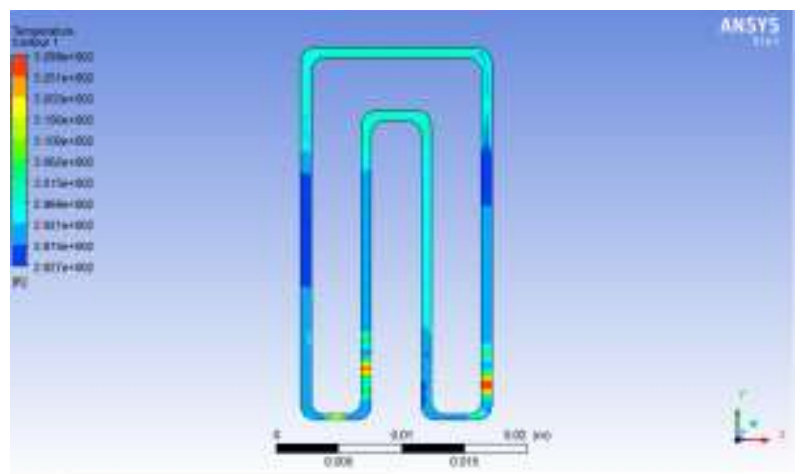

Fig 19. Temperature distribution for $70 \%$

3.3 Three turn MPHP analysis

3.3.1Water liquid volume fraction and temperature distribution for $30 \%$

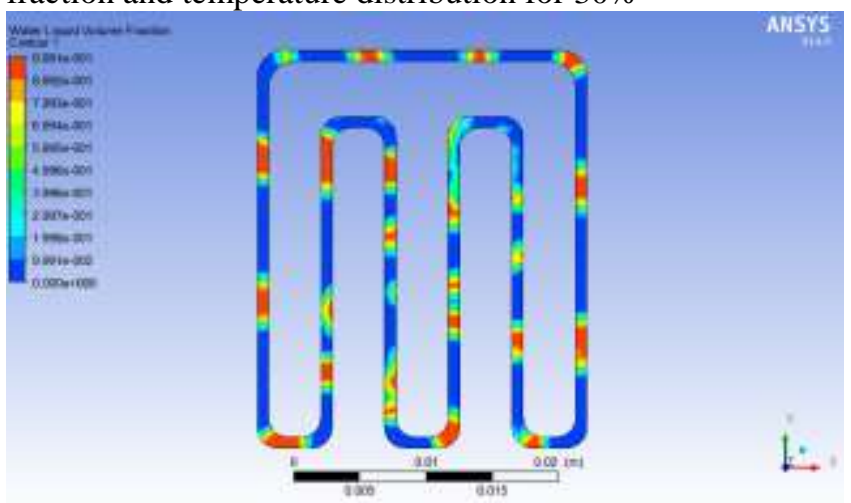

Fig 20. Volume Fraction for 30\%

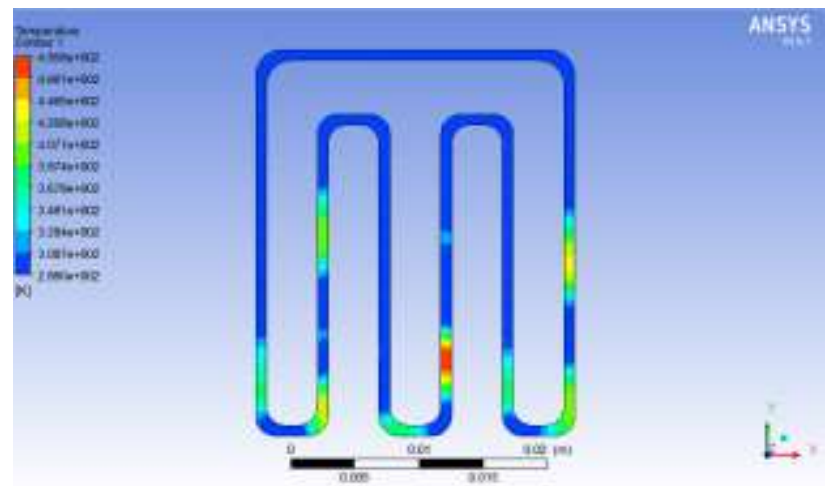

Fig 21. Temperature distribution for 30\%

3.3.2 Water liquid volume fraction and temperature distribution for $50 \%$

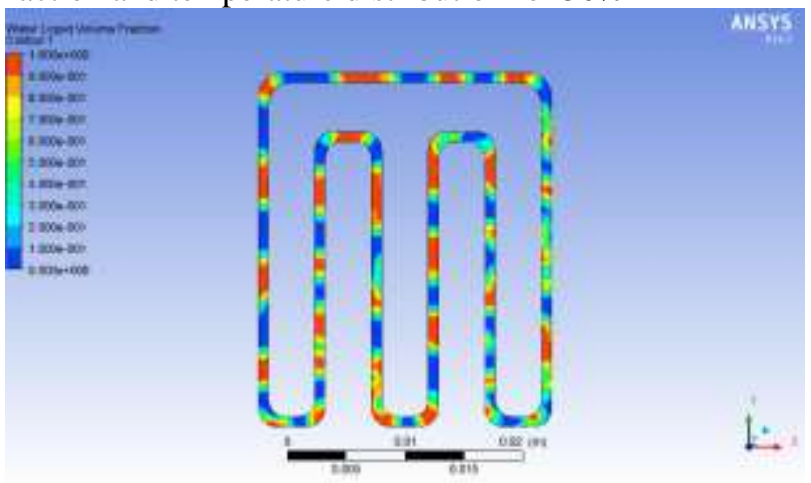

Fig 22. Volume Fraction for $50 \%$ 


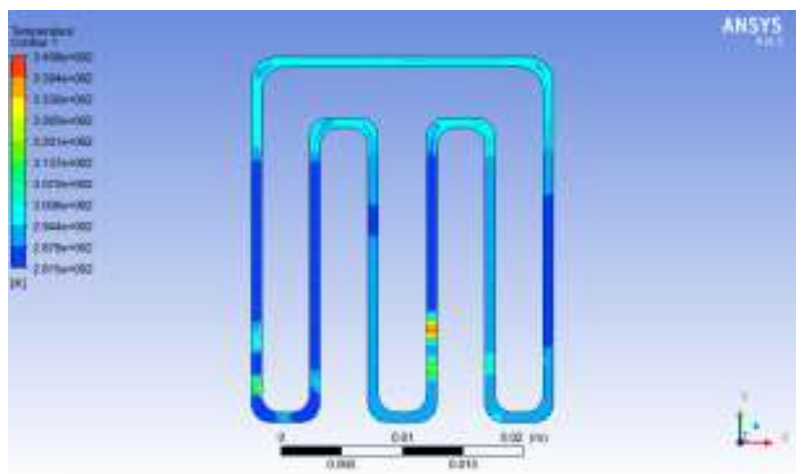

Fig 23. Temperature distribution for $\mathbf{5 0 \%}$

3.3.3 Water liquid volume fraction and temperature distribution for $70 \%$

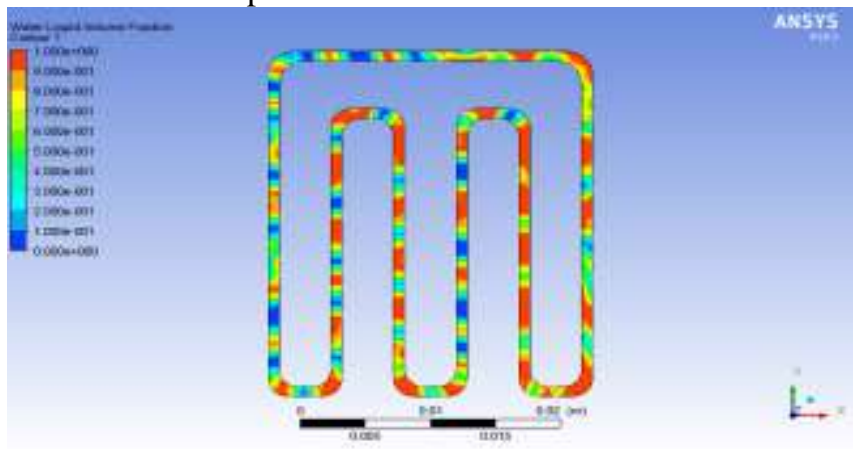

Fig 24. Volume Fraction for $\mathbf{7 0 \%}$

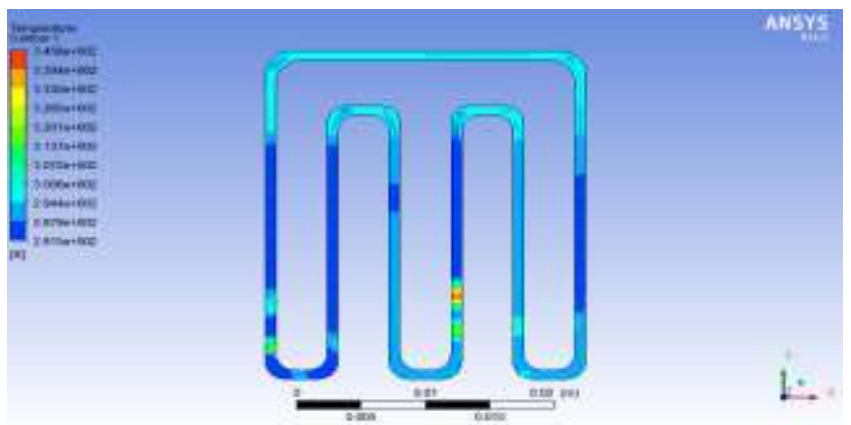

Fig 25. Temperature distribution for $\mathbf{7 0 \%}$

\subsection{Thermal Performance Evaluation}

Using equations (6) and (7) and results obtained from Ansys Fluent thermal performance of different MPHPs was evaluated which is summarized in Table 4 and Table 5.

Table 4. Temperatures of Evaporator section and Condenser Section

\begin{tabular}{|c|c|c|c|c|}
\hline \multirow{2}{*}{ No of turns } & Temperature & $30 \%$ & $50 \%$ & $70 \%$ \\
\hline \multirow{2}{*}{1} & $\mathrm{~T}_{\mathrm{e}}(\mathrm{K})$ & 249.177 & 309.662 & 300.529 \\
\cline { 2 - 5 } & $\mathrm{T}_{\mathrm{c}}(\mathrm{K})$ & 240.901 & 298.636 & 297.645 \\
\hline \multirow{2}{*}{2} & $\mathrm{~T}_{\mathrm{e}}(\mathrm{K})$ & 499.284 & 314.362 & 308.431 \\
\cline { 2 - 5 } & $\mathrm{T}_{\mathrm{c}}(\mathrm{K})$ & 298.7005 & 287.7435 & 295.66 \\
\hline \multirow{2}{*}{3} & $\mathrm{~T}_{\mathrm{e}}(\mathrm{K})$ & 387.834 & 331.31 & 314.803 \\
\cline { 2 - 5 } & $\mathrm{T}_{\mathrm{c}}(\mathrm{K})$ & 298.184 & 298.1005 & 296.1405 \\
\hline
\end{tabular}

Table 5. Thermal Performance results

\begin{tabular}{|c|c|c|c|c|}
\hline No of turns & Thermal Parameters & $30 \%$ & $50 \%$ & $70 \%$ \\
\hline \multirow{2}{*}{1} & $\mathrm{~h}\left(\mathrm{~W} / \mathrm{m}^{2} \mathrm{~K}\right)$ & 121.2606 & 90.6947 & 346.74 \\
\cline { 2 - 4 } & $\mathrm{R}\left(\mathrm{m}^{2} \mathrm{~K} / \mathrm{W}\right)$ & 0.00827 & 0.011026 & 0.002884 \\
\hline \multirow{2}{*}{2} & $\mathrm{~h}\left(\mathrm{~W} / \mathrm{m}^{2} \mathrm{~K}\right)$ & 5.01027 & 37.5671 & 78.3024 \\
\cline { 2 - 5 } & $\mathrm{R}\left(\mathrm{m}^{2} \mathrm{~K} / \mathrm{W}\right)$ & 0.1995 & 0.02662 & 0.012771 \\
\hline \multirow{2}{*}{3} & $\mathrm{~h}\left(\mathrm{~W} / \mathrm{m}^{2} \mathrm{~K}\right)$ & 11.1544 & 30.1118 & 53.5833 \\
\cline { 2 - 5 } & $\mathrm{R}\left(\mathrm{m}^{2} \mathrm{~K} / \mathrm{W}\right)$ & 0.08965 & 0.0332 & 0.01866 \\
\hline
\end{tabular}




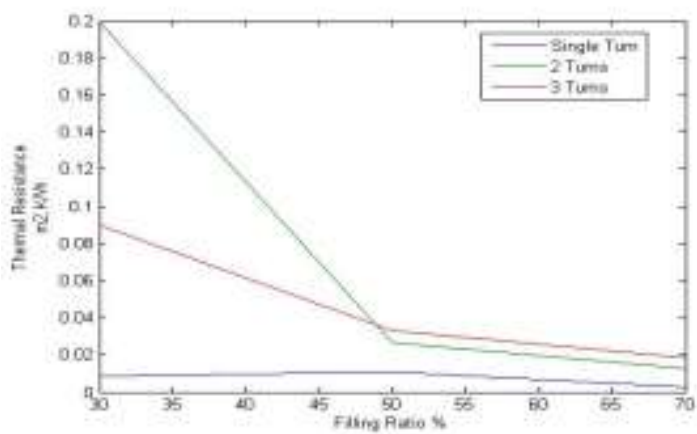

Fig 25. Thermal Resistance variation

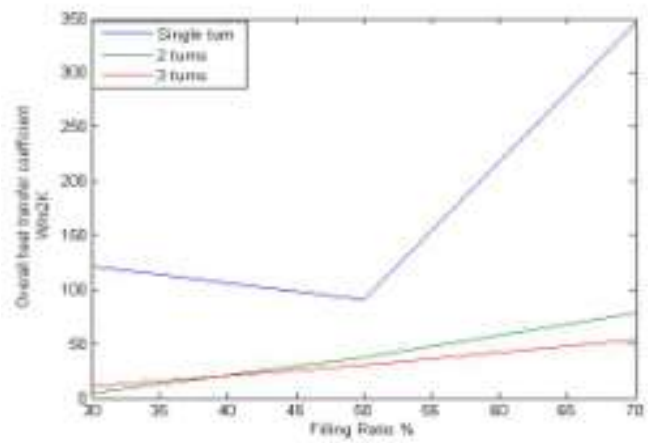

Fig 26. Overall Heat transfer coefficient variation

From the above graphs, it can be summarized that as the filling ratio increases the thermal resistance decreases and the overall heat transfer coefficient increases. This shows that by increasing the filling ratio the performance of the MPHP in terms of heat transfer increases. However, the limit of filling ratio is constrained up to $80 \%$.Because slug formation ceases to exist above this value. We observe that in case of single turn MPHP, the heat transfer coefficient first decreases and then increases abruptly. This behavior is unstable. In case of 2 and 3 turns MPHP, the coefficient increases steadily. By observing the graph of Thermal resistance variation, we find that the resistance of single turns increases first and then decreases later, which is abnormal. In case of 2 turns the resistance is very high at $30 \%$ and drops to minimum value. For 3 turns, the resistance is low for $30 \%$ and decreases with increase in filling ratio. So, it is inferred that out of the three cases, 3 turn MPHP shows more stable behavior as compared to single and 2 turn MPHP.

\section{CONCLUSION}

In this study, The fundamentals of operation of MPHP have been discussed. By considering these fundamentals and design parameters, the simulation analysis has been carried out for 3 different geometries of MPHPs having filling ratios namely $30 \%, 50 \%$ and $70 \%$. It was observed from the study that 3 turn MPHP performs better as compared to the other two cases with variation in the Filling Ratio. This was the conclusion from the study.

\section{REFERENCES}

[1] Mr. Mohd Aijaz ul Haque, Dr. Md Azizuddin, Mr. Md Khalid Rehman. October 2016. CFD and volume fraction analysis of closed loop pulsating heat pipe (CLPHP). IOSR Journal of Mechanical and Civil Engineering.

[2] S. M. Pouryoussefi, Yuwen Zhang. January 2017 Analysis of chaotic flow in a 2D multi-turn closed-loop pulsating heat pipe. Applied Thermal Engineering

[3] Soohwan Jun, Sung Jin Kim. December 2015 Comparison of the thermal performances and flow characteristics between closed loop and closed end micro pulsating heat pipe. International Journal of Heat and Mass Transfer.

[4] Junji Gu, Masahiro Kawaji, Pyosuke Futamata. 2005 Microgravity Performance of Micro Pulsating Heat Pipe. Sci. Technol.

[5] Next Generation Microchannel Heat exchangers by Michael M. Ohadi, Kyosung Choo, Serguei Tessiatoun. Page number 95 to 105.

[6] Umberto Costa Bitencourt, July 2016, CFD Simulation of a Pulsating Heat Pipe using Ansys Fluent. Research gate.

Malay S. Patel. " Comparison of Heat Transfer rate of closed loop micro pulsating heat pipes having different number of turns " The International Journal of Engineering and Science

(The IJES) 6.7 (2017): 01-12 